\title{
Barcelona, caminando hacia la resiliencia urbana en el barrio de Vallcarca
}

\author{
Barcelona: heading towards urban resilience \\ in the Vallcarca neighbourhood
}

\author{
Rafael de Balanzo Joue* \\ Fecha de recepción: 21-05-2014 - Fecha de aceptación: 21-11-2014 \\ Hábitat y Sociedad (ISSN 2173-125X), n. ${ }^{\circ} 8$, noviembre de 2015, pp. 75-95.
}

\section{Summary}

Many neighbourhoods and cities are succumbing to multiple and successive changes caused by internal collapse and external systemic crises. The neighbourhood of Vallcarca in the Gràcia district has become an urban landmark of the city of Barcelona. It is characterized equally by belonging to one of the first districts of Barcelona to implement policies of urban sustainability, and by succumbing for more than a decade to a process of crisis, devastation and conflict induced by a development plan based on the demolition/new construction precept.

This research uses the adaptive cycle heuristic and the theory of panarchy (Holling and Gunderson, 2002) to identify the development of resilience in the dynamics of urban practices in the neighbourhood from a new systemic, evolutionary and multi-scale vision used for the study of socio-ecological systems in the field of ecology, and of governance policies. This vision has, as yet, been only minimally applied in urban environments. Furthermore, it is a given fact that research has been conducted through the participant observation methodology throughout the urbanization process of the last 12 years.

The resilience construction process of the Vallcarca district starts from a first, predictable, and institutionalized long period of impoverishment with rigid urban planning of inflexible development. The district takes advantage of a window of opportunity for change and initiates a second stage of revival and innovative reorganization to build resilience. This process materializes thanks to relationships of memory and a return to panarchy (Gunderson, 2010) and to inter-scalar and extra-scalar relations of the agents and transformational

\section{Resumen}

Multitud de barrios y ciudades están sucumbiendo a múltiples y sucesivos cambios provocados por colapsos internos y crisis sistémicas externas. El barrio de Vallcarca del distrito de Gràcia se ha convertido en un referente urbano de la ciudad de Barcelona. Se caracteriza por pertenecer a uno de los distritos de la ciudad de Barcelona más precursores en la aplicación de políticas de sostenibilidad urbana, y a la par sucumbir en un proceso de crisis, devastación y conflicto inducido por un plan urbanístico basado en el precepto demolición/ nueva construcción desde hace más de una década.

Esta investigación utiliza la heurística del ciclo adaptativo y la teoría de la panarquía (Holling y Gunderson, 2002) para identificar la evolución de la resiliencia en las dinámicas de las prácticas urbanísticas del barrio a partir de una novedosa visión sistémica, evolutiva y multiescalar utilizada para el estudio de los sistemas socioecológicos en el campo de la ecología, y de las políticas de gobernanza, pero mínimamente aplicada en los entornos urbanos hasta ahora. Además, se da el hecho de que se ha podido realizar la investigación a través de la metodología de la observación participante a lo largo del proceso urbanístico en los últimos 12 años.

El proceso de construcción de la resiliencia del barrio de Vallcarca se inicia a partir de una primera, previsible, larga e institucionalizada etapa de empobrecimiento con un planeamiento urbanístico rígido, de desarrollo urbanizador e inflexible. El barrio aprovecha una ventana de oportunidad para el cambio e inicia una segunda etapa de relanzamiento y reorganización innovadora para construir la resiliencia. Este proceso se materializa gracias a las relaciones de memoria y revuelta de la panarquía (Gunderson, 2010) y a las relaciones

\footnotetext{
* Arquitecto, activista urbano, profesor en City University New York, Queens College. Director del Work Programme “Actions Without Borders" de la Unión Internacional de Arquitectos, y doctorando del Instituto de Sostenibilidad, Universidad Politécnica de Cataluña, Barcelona Tech.
} 
institutions (Westley et al., 2013). The attributes of selforganization, adaptability, diversity, learning, and innovation are highlighted as key strategies for adaptive and resilient management of habitat.

\section{Key words}

Urban Resilience, Sustainability, Habitat transformation, Barcelona, Complexity, Panarchy, Adaptive cycle, Change interescalares y extraescalares de los agentes e instituciones transformacionales (Westley et al., 2013). En la parte final, se resalta cómo los atributos de la auto-organización, la adaptabilidad, la diversidad, el aprendizaje y la innovación son estrategias claves para la gestión adaptativa y resiliente del hábitat.

\section{Pallabras clave}

Resiliencia urbana, Sostenibilidad, Transformación del hábitat, Barcelona, Complejidad, Panarquía, Ciclo adaptativo, Cambio

\section{Introducción: relación entre crisis y resiliencia}

Las cuestiones planteadas son: ¿Cómo se adapta y se transforma un barrio de forma sostenible frente a las crisis sistémicas? ¿Qué lecciones nos aporta la experiencia del barrio de Vallcarca? Y ¿qué principales atributos de la resiliencia urbana refuerzan la gestión social del hábitat?

Para afrontar esta situación generalizada de crisis en las ciudades y barrios y entender la compleja perspectiva del hábitat (De Manuel, 2010), se escoge analizar el barrio de Vallcarca de la ciudad de Barcelona y aplicar la analogía de la resiliencia de los sistemas socialecológicos, proveniente de la disciplina de la ecología, y que se denomina ciclo adaptativo para los sistemas socioecológicos (Walker et al. 2004). La ciudad de Barcelona se ha convertido en un referente de la sostenibilidad urbana, ejemplar por las políticas urbanas implementadas en la

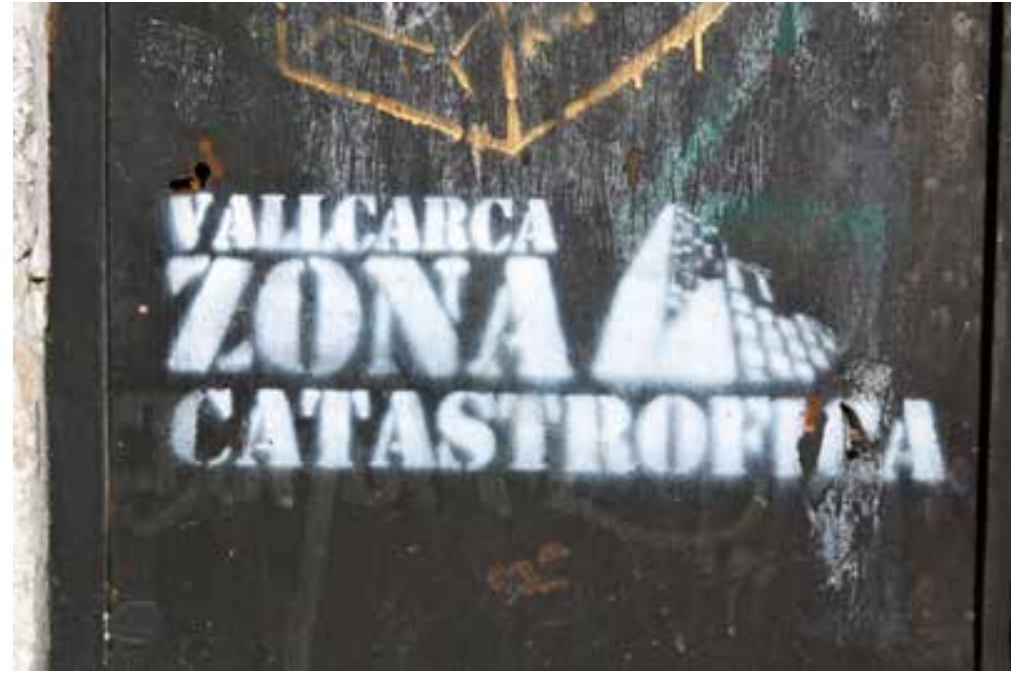
ciudad desde 1996 bajo la Regidoría de la Ciudad Sostenible, iniciativa del por entonces regidor Josep Puig perteneciente al movimiento "Nuclear, No, gracias". Estas políticas de sostenibilidad urbana se focalizan sobre todo en el distrito de Gràcia, pionero en Barcelona. En este contexto favorable de transformación positiva, se encuentra el barrio de Vallcarca, afectado por un plan urbanístico regido por la gestión del suelo a partir del modelo neoliberal (Arico, Stanchieri, 2013), por lo cual el barrio se sumerge en una profunda y constante crisis de más de dos décadas (Figura 1). La metodología utilizada para el análisis de la evolución de las dinámicas de las prácticas urbanísticas en el barrio se fundamenta en:

Figura 1. Grafiti en el barrio de Vallcarca. Fuente: Rafael de Balanzo.
- Aplicar la heurística del ciclo adaptativo y su representación panárquica.

- Poner de relieve la estructura jerárquica de los ciclos adaptativos anidados e interrelacionados reforzados por las relaciones de memoria y revuelta.

- Resaltar los atributos claves de la construcción de la resiliencia en las pequeñas comunidades locales y, en particular, el rol clave de los agentes transformacionales. 
El caso empírico del barrio de Vallcarca se analiza en base a la observación participante desarrollada por el propio investigador como miembro de diversos colectivos del barrio, como la Plataforma Salvem Vallcarca, la Cooperativa Valldures, la Asociación Amics Bosc Turull y La Asamblea Vallcarca en el transcurso de los últimos 12 años. A partir de un primer análisis realizado sobre la evolución de la ciudad de Barcelona en los últimos 50 años con la heurística del ciclo adaptativo y la teoría de la panarquía, se profundiza en el caso del barrio de Vallcarca con la descripción de las principales etapas evolutivas desde la fase de preparación para el cambio, pasando por el hito de la ventana de oportuni$d a d$, hasta la etapa de relanzamiento y de reorganización del barrio hacia la construcción de la resiliencia (Olsson et al. 2006).

\subsection{Lectura de la sostenibilidad urbana desde la resiliencia}

La evolución de la calidad de vida en las ciudades actuales se relaciona con fenómenos como la sobrepoblación, el cambio climático y la inmigración, provocados por las diferentes crisis sistémicas medioambientales, económicas, sociales y políticas que afectan la gobernanza de las ciudades. Estos colapsos de corta y larga duración afectan la sostenibilidad de los sistemas urbanos, provocando importantes cambios sustanciales. Consecuentemente, se requiere reorientar las políticas urbanas de planificación sostenible hacia nuevas estrategias de adaptación y transformación vía procesos más resilientes.

Actualmente, las estrategias urbanas tipo top-down (de arriba abajo), como el caso de Barcelona, están dirigidas principalmente por instituciones municipales que se basan en reforzar la eficiencia metabólica de la ciudad contemporánea mediante la monopolización tecnológica con las llamadas Ciudades Inteligentes (Smart Cities) y reducir la vulnerabilidad de las infraestructuras y de sus ecosistemas frente a las catástrofes (Engineering Resilience). Junto a estas estrategias institucionales topdown se desarrollan en paralelo estrategias urbanas tipo bottom-up (de abajo arriba). Dichas estrategias están promovidas y autogestionadas por colectivos urbanos locales que pretenden motivar la sociedad para adaptarse y transformarse ante los cambios generados por la crisis sistémica a partir del concepto de la resiliencia evolutiva.

La etimología de la palabra resiliencia procede del concepto resilio, compuesto por el prefijo re y el verbo salire, saltar, significando algo así como volver de un salto (Fernández, Morán, 2012). La resiliencia se define como la capacidad de un sistema para absorber las perturbaciones y reorganizarse mientras se somete a un cambio, conservando esencialmente la misma función, estructura, identidad y retroalimentación (Walker et al., 2004). Esta definición se utiliza comola metáfora de la "Ciudad Resiliente" (Pickett et al., 2004). No obstante, se utilizará un segundo enfoque que define la resiliencia como la capacidad de adaptación de los sistemas socio-ecológicos (a partir de ahora, SSE) a los cambios y colapsos (Folke, 2006). Un SSE puede definirse como la vinculación e interrelación entre un sistema ecológico y los sistemas sociales existentes en un entorno definido. Esto implica que las ciudades son sistemas complejos adaptativos (Portugali, 2012), capaces de autoregularse y fomentar soluciones innovadoras para el desarrollo urbano. Esta visión se aplica, por ejemplo, en el movimiento de Ciudades en Transición (Transition Towns) (Hopkins, 2008, 2011) y forma parte de las estrategias bottom-up. Cada iniciativa de Ciudades en Transición tie- 
ne en cuenta cómo la sociedad puede superar un colapso energético, ya que las comunidades locales carecen de la capacidad de adaptación (Hopkins, 2008). Por tanto, se constata que se está planteando, por parte de diversos agentes urbanos, la reorientación de las políticas urbanas actuales a partir del concepto de la resiliencia con una visión sistémica y evolutiva a todas las escalas temporales y espaciales de la disciplina del urbanismo de las ciudades.

\subsection{Elementos claves de la resiliencia urbana evolutiva}

La sostenibilidad de las ciudades no está solo intrínsecamente relacionada con la resiliencia a corto plazo frente a las grandes catástrofes, sino también con la resiliencia a largo plazo para adaptarse y transformarse a partir del aprendizaje evolutivo aportado por los cambios sociales, económicos, culturales y medioambientales. El desarrollo sostenible de los sistemas urbanos se basa en el grado de adaptabilidad y de transformabilidad a los cambios sistémicos. Dicho grado es lo que denominamos el concepto evolutivo de la resiliencia (Davoudi, 2013). Este concepto, denominado resiliencia ecológica o resiliencia evolutiva se basa en diferentes etapas de cambios (stress permanentes), y a la imprevisibilidad de los ciclos adaptativos de los SSE, y tiene su origen en la perspectiva ecológica planteada por Holling $(1973,1986)$ que se aplica a los sistemas SSE (Holling, 2001, y Walker et al. 2012). La definición de la resiliencia de un SSE contiene las siguientes características (Holling et al. 2002):

- La cantidad de cambios que un sistema puede sufrir y seguir conservando esencialmente la misma función, estructura e identidad.

- El grado de capacidad de autoorganización del sistema socioecológico.

- La oportunidad del sistema socioecológico para construir y aumentar la capacidad de aprendizaje y adaptación.

El ciclo de renovación adaptativa nos muestra, por un lado, la etapa hacia detrás (Back Loop), donde las fuerzas desestabilizadoras son un factor importante para mantener la diversidad, la resiliencia y la oportunidad. Estos factores son importantes para la innovación. Por otro lado, la etapa hacia adelante (Front Loop) donde las fuerzas estabilizadoras son también básicas para mantener la productividad del sistema, y su crecimiento que una vez reorganizado mediante las relaciones de memoria y revuelta establece un desarrollo sostenible (Figura 2). Olsson et al. (2006) utiliza y reinterpreta el ciclo adaptativo como un instrumento de análisis de los SSE incluyendo el factor humano para fomentar la resiliencia. Olsson et al. (2006) interpreta el ciclo adaptativo a partir de las fases de preparación para el cambio marcado por el hito de la ventana de oportunidad, seguido de la fase de navegando por la transición, y finalmente, de la fase de construcción de la resiliencia. La presencia del componente humano confiere la especificidad de un SSE con las tres características siguientes, según Holling (2001): 1) la capacidad prospectiva; 2) la capacidad de anticipación; y 3) la intencionalidad en las acciones de los humanos que pueden reducir considerablemente el impacto de los cambios en un sistema. La resiliencia social es definida como la habilidad de la comunidad de personas para resistir y recuperarse de choques y perturbaciones (Adger, 2000). Según argumentan Holling et al. (1996), los SSE no solo pueden ser gobernados por una gestión 


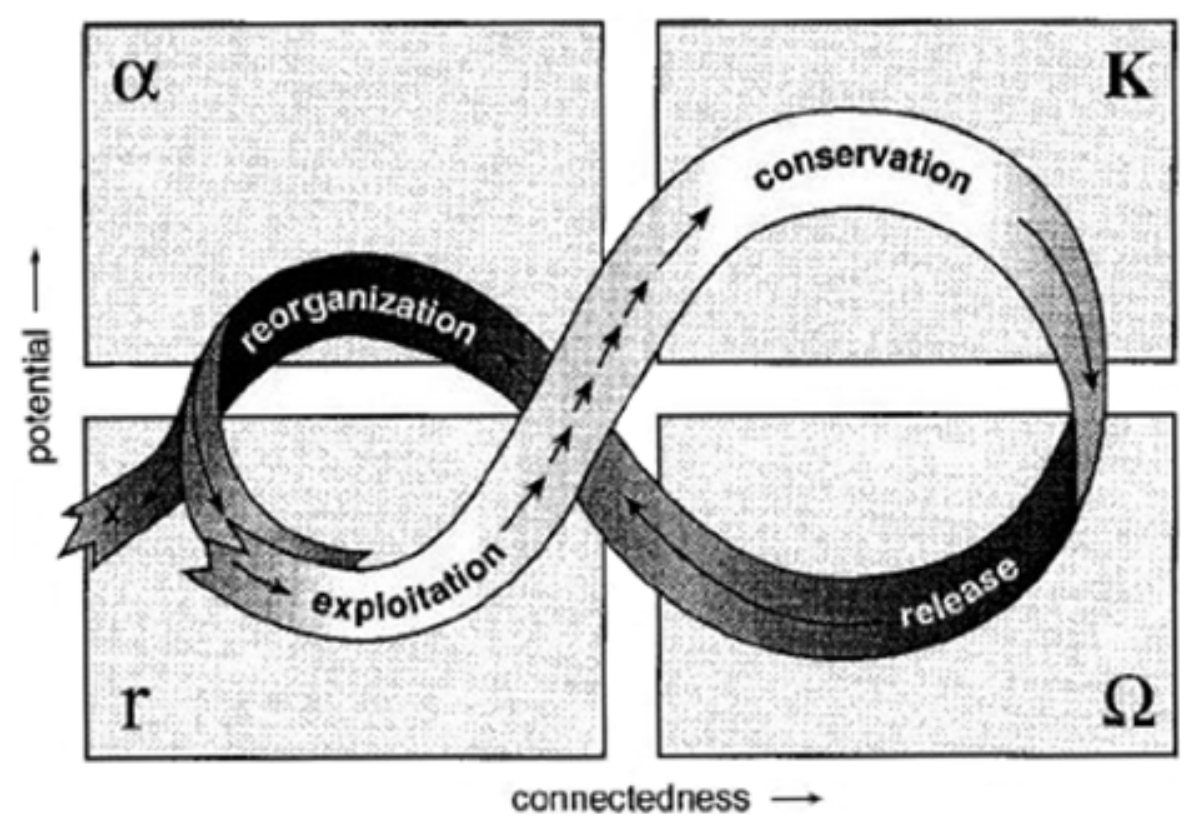

top-down asociada a veces con ideas convencionales de liderazgo, sino que también es necesario un liderazgo transformacional por parte de las pequeñas comunidades locales para la sostenibilidad de los SSE. La resiliencia de los SSE se basa en una sociedad flexible y capaz de ajustarse a un contexto de incertidumbre y cambios, y capaz de aprovechar las oportunidades positivas que el futuro pueda traer (Folke, 2003).

\subsection{Relación entre panarquía y barrio}

La panarquía es un concepto que proviene de la teoría de la resiliencia y permite una interpretación más transdisciplinar y holística de la transformación y evolución de los SSE sometidos a recurrentes cambios sociales, medioambientales, y económicos (Holling et al. 2002). El prefijo Pan proviene del griego y significa totalidad, y la panarquía es la representación jerárquica de un conjunto de SSE entrelazados de ciclos adaptativos (Holling, 2001). Para introducir las diferentes escalas de los SSE y sus interacciones, se utiliza la escala espacial y temporal. En ella se representan dos aspectos: en ordenadas el ámbito espacial de cada ciclo adaptativo y en abscisas el ámbito temporal en el que transcurren las etapas de cambio. Como se puede ver en la Figura 3, los niveles superiores de la panarquía son más lentos y tienen un ámbito espacial mayor que los niveles inferiores, que son rápidos y corresponden a espacios más reducidos. Un ciclo adaptativo de referencia depende escalarmente de la retroalimentación e influencia de los ciclos adaptativos inferiores y superiores y, por todo ello, el ciclo adaptativo no se puede explicar sin tener en cuenta las fuerzas que logran un equilibrio dinámico de los sistemas y que actúan a través de las escalas mediante las relaciones panárquicas de Memoria o Revuelta (Holling et al., 2002). Por tanto, las actuales políticas urbanas y sus modelos de planeamiento urbano de-
Figura 2. Fuente: Holling et al., 2002 El ciclo adaptativo se representa con cuatro fases simbolizadas por los siguientes factores: Omega: Inestabilidad colapso, destrucción creativa, cambios constante, interactividad baja; Alpha: Movilización, reorganización, regeneración, redefinición, flexibilidad del sistema; r: Explotación y crecimiento, diversidad y conectividad, disponibilidad de recursos; y K: Conservación y estabilidad, organización fuerte, rigidez y cambios lentos.

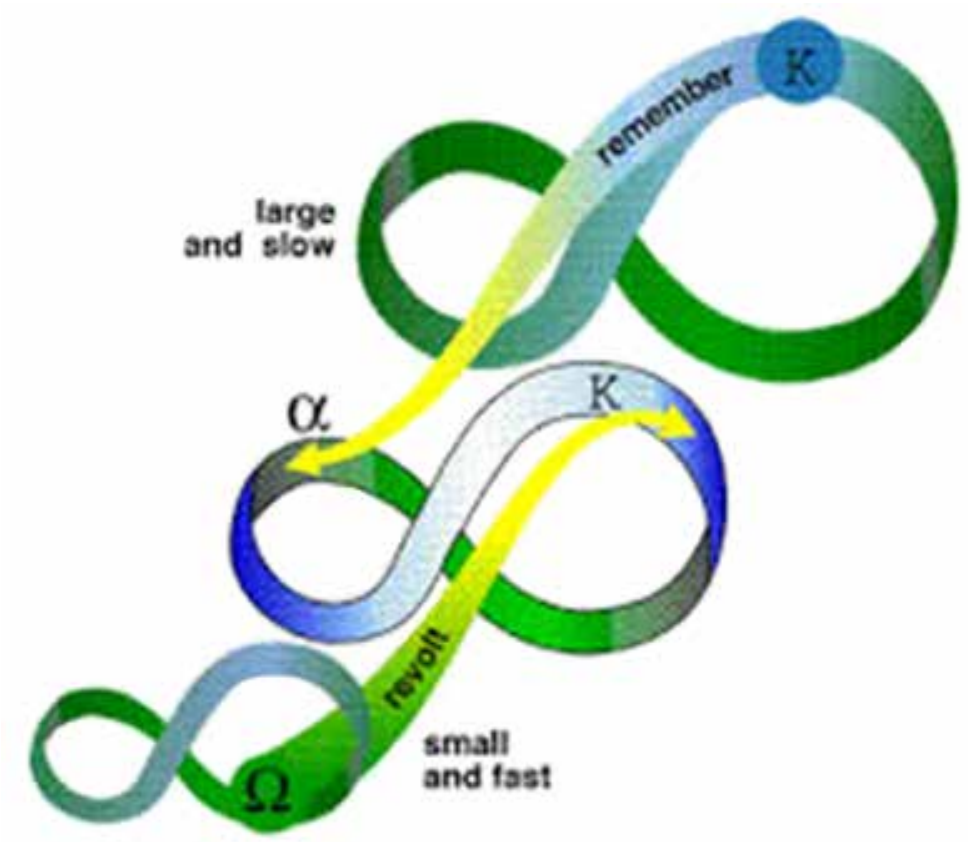


Figura 4. Lectura del contexto urbano de la Panarquía. Fuente: Elaboración propia según Holling et al. (2002).

\section{Three nested systems}

PANARCHY CONTEXT

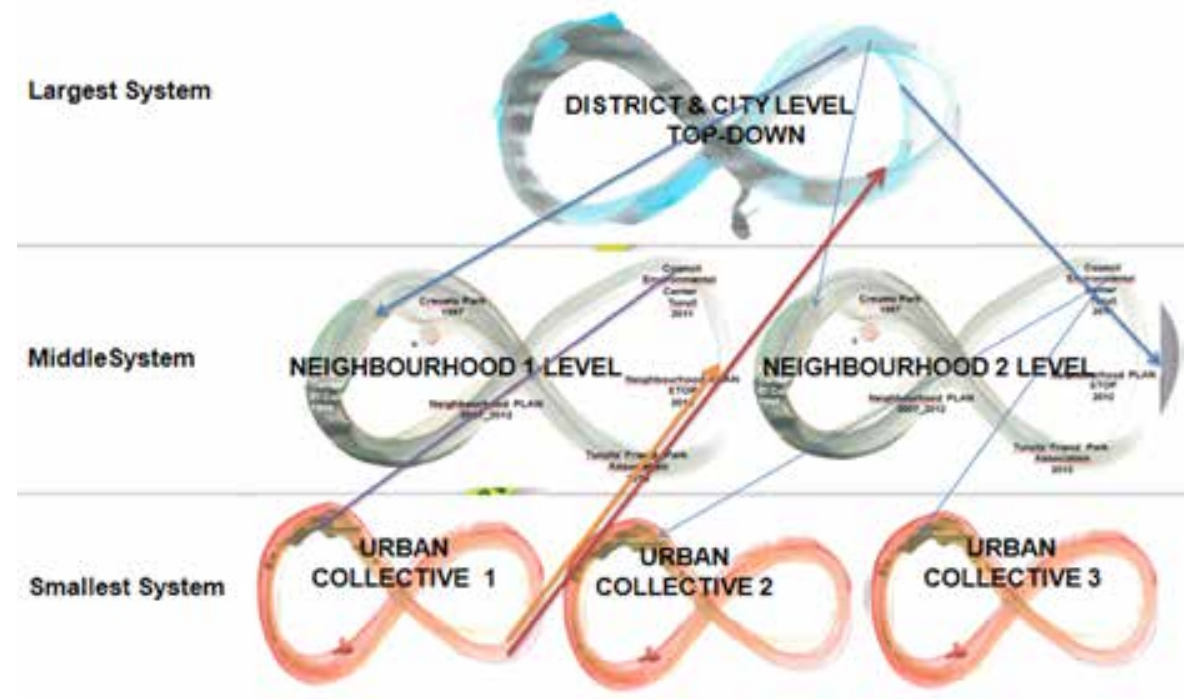

ben incluir esta visión panárquica de la ciudad y es necesario desarrollar este concepto como herramienta para el análisis y desarrollo de las políticas urbanas sostenibles. Para analizar el caso práctico del barrio de Vallcarca se utiliza el concepto de representación de la panarquía en una perspectiva multiescalar que está integrada por tres niveles de los SSE (Ciudad, Distrito y Barrio) con sus respectivos ciclos adaptativos en las diferentes escalas del espacio, tiempo y de organización (Figura 4).

\section{Barcelona y la heurística del ciclo adaptativo}

Se trata de ejemplificar la aplicación de la heurística del ciclo adaptativo como modelo de análisis de los cambios en las dinámicas urbanas en un caso práctico, validarlo como modelo para poner de relieve las interacciones existentes a diferentes escalas de la panarquía en base a los efectos de memoria y de revuelta de las relaciones interescalares y extraescalares, para destacar el factor clave de los agentes transformacionales y para distinguir los atributos específicos de la resiliencia. Como punto de partida, la ciudad de Barcelona ha demostrado que se erige como un caso único de gran relevancia en el ámbito del urbanismo en las últimas décadas. En primer lugar, el Modelo Barcelona es reconocido internacionalmente por la actuación en el espacio público y la creación de equipamientos para la regeneración urbana de la ciudad durante la década de los ochenta y principios de los noventa (Borja, 2009; Busquets, 2005; Montaner, 2004). En segundo lugar, Barcelona se sitúa como una de las ciudades pioneras en España en la implantación de políticas de sostenibilidad urbana con la creación del área de la Ciudad Sostenible en 1996. A continuación, se propone analizar la ciudad de Barcelona según la heurística de ciclo adaptativo (Holling et al., 2002) y la teoría de la panarquía para reflejar la evolución de las dinámicas urbanas ante los cambios y crisis. Este análisis evidencia que la ciudad de Barcelona durante las últimas décadas ha desarrollado una sola y corta etapa de renovación creativa, innovación y reorganización (de 1974 a 1980) en las prácticas urbanísticas. 
- El período de la Vivienda se inicia a principios de los años cincuenta y está caracterizado por la necesidad de producción de hábitat urbano en un proceso urbanizador desenfrenado ante la necesidad imperiosa de vivienda provocado por los movimientos migratorios del campo a la ciudad industrializada (Fernández Durán, 2006). La consecuente crisis y colapso de la ciudad están provocados por los movimientos sociales urbanos ante el trauma económico, social, medioambiental y político por la falta de equipamientos sanitarios, culturales, educativos y, ante todo, por la falta de libertades democráticas. La llegada de la democracia y la propuesta de cambio en el planeamiento de la ciudad (revisión del Plan Comarcal del 53 a partir de 1974) configuran una ventana de oportunidad para el cambio con la revuelta de los movimientos sociales urbanos que están preparados para el cambio. La fase de navegar por la transición se inicia con innovadoras prácticas urbanísticas como los planes populares y otras múltiples acciones ciudadanas como exposiciones, concursos, talleres, etc.

- Desde 1979 a 1994 se desarrolla un excepcional período simbolizado por el concepto del proyecto urbano por parte del nuevo Servicio de Urbanismo dirigido por el arquitecto Bohigas. Esta etapa de renovación creativa y reorganización multiescalar simultánea a escala del barrio y de las instituciones municipales de la ciudad será breve y no se consolida. En primer lugar, el proceso de cogestión en las prácticas urbanísticas se diluye con el traspaso de la mayoría de los agentes transformacionales (técnicos y políticos) y de las cédulas políticas clandestinas como redes sombra (Olsson et al., 2006) a los puestos de la administración pública (FAVB, 2009). En segundo lugar, se vuelve a imponer la memoria del modelo de gestión urbanística top-down del período anterior. Este período paradigmático de la gestión del urbanismo barcelonés, fuertemente tecnocrático y dirigista, no se inició en los ayuntamientos elegidos democráticamente, sino que proviene de la memoria que está prefigurada en el Plan de 1953, más todavía en su revisión de 1964, y de una forma definitiva y vigente en el Plan General Metropolitano (PGM) de 1976 (Delgado, 2007). Esta etapa se denomina de crecimiento e innovación creativa institucional. A finales de los años ochenta empezaron a oírse voces críticas sobre las políticas municipales en relación a los diversos frentes de actuación (Capel, 2005). La llegada del partido Alternativa-Verdes en el Gobierno municipal, permite el inicio del siguiente período con las políticas urbanas para la sostenibilidad, sin que el período anterior del proyecto urbano haya entrado en crisis.

- El período anterior del proyecto urbano se actualiza a partir de 1995 hasta la actualidad con los conceptos de la Eficiencia y la Sostenibilidad, por la continuidad de la gestión top-down, la aclimatación a las dinámicas globalizadoras internacionales, con planteamientos más aleatorios, y con el interés de la promoción inmobiliaria internacional privada en invertir en la marca Barcelona (Delgado, 2007). La elaboración y gestión de la Agenda 21 local de Barcelona tampoco incorpora la cogestión ciudadana y empresarial en su proceso de desarrollo ni tampoco determina los hitos necesarios para implementar las líneas estratégicas de la Agenda 21 según propone la Guía básica de planificación de la Agenda 21 local de Naciones Unidas acordada en la Cumbre de 
Río de 1992 (Entrevista realizada a Pep Puig, 2014). Asistimos a la llegada en Barcelona de una fase de diferentes crisis y colapsos por la falte de los suministros básicos como el agua potable y la energía, por la falta de la protección del patrimonio cultural como Can Ricart (Capel, 2010), por la falta de innovación en la gobernanza como el referéndum de la reforma de la Diagonal y el proyecto del Forat de la Vergonya, pero sobre todo por la falta de vivienda pública. Este momento de confusión lleva a la ciudad hacia una nueva fase de preparación para el cambio promovida por los nuevos movimientos sociales empezando por Repensar Barcelona hasta las Asambleas 15-M de los barrios. Aparece entonces una ventana de oportunidad que se inicia con la gestión ciudadana de los equipamientos públicos (Harmonía, Orlandai, Turull, etc.) y de los espacios públicos con el Plan BUITS (Vacíos urbanos con implicación territorial y social) del Ayuntamiento de Barcelona.

\section{Vallcarca, barrio incubadora de resiliencia}

A continuación se analiza la evolución multiescalar de la prácticas urbanísticas en el barrio de Vallcarca (Figura 5) respecto al distrito de Gràcia y a la ciudad de Barcelona. El barrio, situado en una zona angosta del valle, se caracteriza por ser el paso natural de numerosas infraestructuras urbanas a lo largo de los siglos, desde los caminos rurales, las explotaciones acuíferas, las canteras de piedra, las autovías urbanas, el metro, para llegar a los proyectos de la ciudad sostenible con los llamados corredores verdes propuestos en el concurso de las 16 puertas de Collserola y para el futuro Parque de Tres Turons. El barrio está expuesto a diferentes cambios urbanísticos y sujeto a la Modificación del Plan General Metropolitano del año 2002 presentada por los Servicios de Urbanismo del Ayuntamiento de Barcelona. Este nuevo plan de gestión del suelo, definido posteriormente con un plan de renovación urbana (PMU, 2008, Plan de Mejora Urbana), está concebido para ser liderado por promotores privados. Este proceso de gentrificación que implica derribar los barrios de baja densidad y de clase trabajadora y

Figura 5. Emplazamiento del distrito de Grácia y del barrio de Vallcarca.

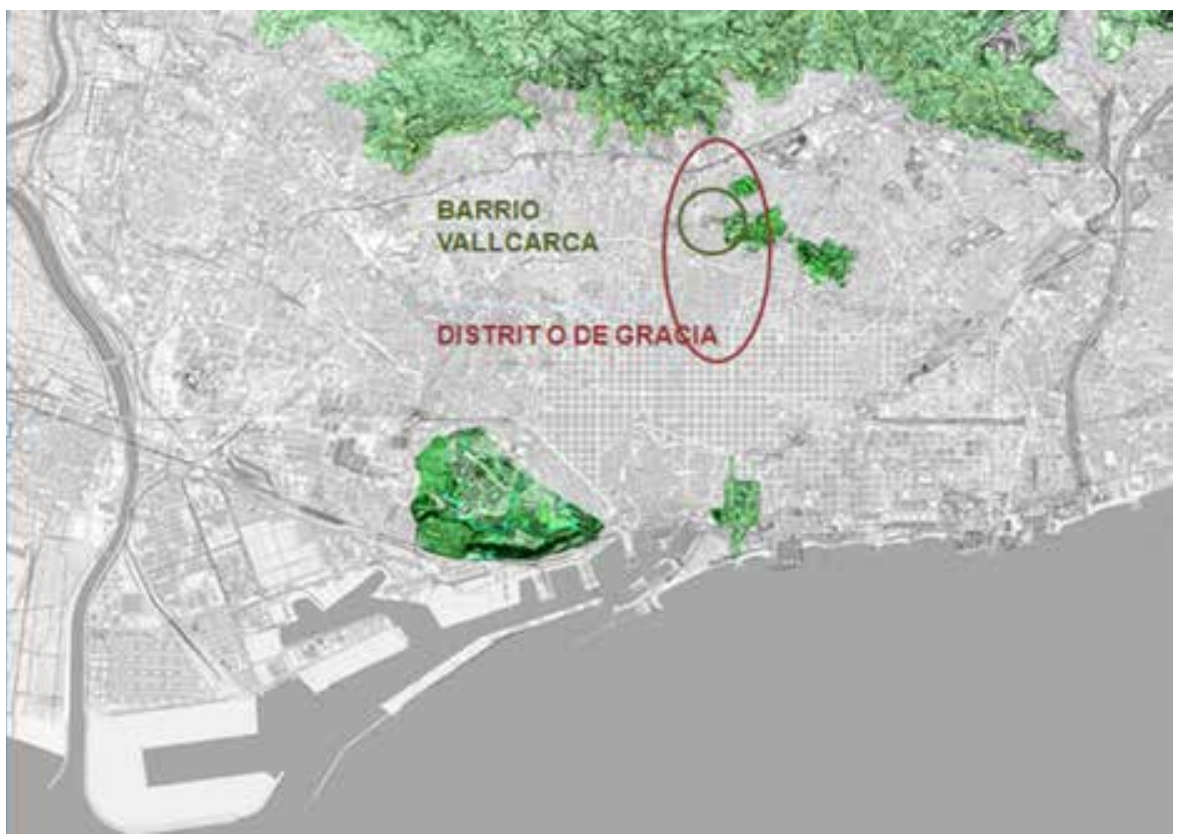


crear en su lugar una zona residencial, se convierte en el concepto urbano más extendido de la ciudad contemporánea (Less et al., 2013).

Los vecinos y el movimiento okupa existente desde 1996 en el barrio de Vallcarca inician un frente común contra el plan urbanístico aprobado en el año 2003 y crean un huerto urbano. Con la llegada de la crisis actual económica, social y medioambiental, el Ayuntamiento de la ciudad de Barcelona, el distrito de Gràcia y los promotores privados quedan atrapados por el plan urbanístico económicamente inviable. La llegada del movimiento 15-M en Barcelona contagia las iniciativas del barrio de Vallcarca, y se inicia una nueva fase de preparación para el cambio en las prácticas urbanísticas. Los nuevos colectivos urbanos y los líderes transformacionales ubicados en las diferentes escalas de la panarquía del barrio serán referentes en la construcción de la resiliencia del barrio.

\subsection{Vallcarca, un barrio sin memoria reivindicativa}

Para profundizar en el proceso de construcción de la resiliencia de la escala pequeña del barrio se utiliza la heurística del ciclo adaptativo y la teoría de panarquía según los períodos establecidos por los ciclos adaptativos de la escala superior (Ayuntamiento de Barcelona) y se resaltan las interacciones interescalares de revuelta y memoria.

El periodo de la Vivienda significa en Vallcarca la transformación de la zona poco habitada, mal comunicada y topográficamente compleja de los cerros en una de las zonas de viviendas de autoconstrucción y barracas (Tatjer, 2010). También se desarrollan nuevas actividades industriales como la explotación de los recursos naturales característicos y simbólicos del territorio de Vallcarca con la extracción de la piedra y del agua potable. Hasta los años setenta, la conexión principal del barrio de Vallcarca con el centro de la ciudad de Barcelona es muy precaria y solo se realizaba mediante el tranvía que ascendía por la calle Mare de Deu del Coll. En la parte central del barrio se estableció toda una red de más de 30 pequeños negocios de alimentación (horno de pan, pescadería, vaquería, colmados, etc.), y oficios relacionados con la construcción y el mantenimiento de edificios (carpinterías, ferreterías, lampisterías y otros) que facilitan los servicios al barrio, y a las zonas más alejadas, sin olvidar la existencia de dos escuelas.

A medida que se redacta y se define el PGM de 1976 se decreta el casco urbano de Vallcarca como SRI, ${ }^{1}$ Sector de Reforma Interior, pendiente de futuras modificaciones para mejorar la situación urbana estratégica desde el punto de vista de la movilidad urbana e interurbana con vehículos privados y convertirse en un área privilegiada, limítrofe al futuro Parque de Tres Turons que comprende 120 ha. Los vecinos de Vallcarca, debido a la poca densidad de población y a su situación de estabilidad provisional, no participan en la gran movilización vecinal de Barcelona de los setenta en contraposición a los barrios reivindicativos colindantes como El Coll y Lesseps con acciones emergentes e innovadoras como los Planes Populares, exposiciones y debates.

El período siguiente del Espacio público y de los Equipamientos desde 1980 a 2002 se inicia en el distrito de Gràcia, con diferentes proyectos urbanos concentrados en los espacios públicos claves como las plazas emblemáticas de la parte central de la Vila de Gracia, con la consecuente creación de parkings subterráneos, y en la creación del Parque de la Creueta en la antigua cantera del barrio del Coll. El barrio de Vallcarca, al ser un barrio de baja densidad y sin reivindicaciones vecinales,
1 Este objetivo se realizó a partir de la delimitación de los Sectores de Reforma Interior (SRI) sujetos al Plan Especial Previo Obligatorio (PERI) en zonas denominadas de «remodelación» (de carácter público o privado). La definición de estos sectores afecta sobre todo a industrias obsoletas, pero también en otros casos afecta a actividades en funcionamiento, sometidas a fuertes presiones urbanas por su posición en la ciudad. Ver en Amador Ferrer (1997): El Pla General Metropolità de Barcelona. La versión de 1976. Papers. Regió Metropolitana de Barcelona, 28, pp. 43-54, noviembre, 1997. Recuperado el 15 de septiembre de 2015, de: http://www.raco. cat/index.php/PapersIERMB/article/ viewFile/102594/128683. 
no se beneficia del proyecto urbano (Entrevista a J. M. Llop, 2014), ni tampoco se desarrolla su Plan Especial de Reforma Interior (PERI). La dinámica de las prácticas urbanísticas de este período se ciñe exclusivamente a los barrios con reivindicaciones vecinales.

\subsection{La metáfora del planeamiento urbano}

El tercer período de la Eficiencia y Sostenibilidad se inicia a partir de 2002 en el distrito de Gràcia con la implantación del Plan Integral de Movilidad. El plan desencadena nuevas prácticas participativas ciudadanas lideradas por el mismo regidor del distrito, Ricard Martínez (educador social), así como por el equipo interdisciplinar de técnicos compuesto por la gerente, arquitectos, ingenieros y educadores sociales. Esta experiencia innovadora fomenta a posteriori el lanzamiento del Plan Comunitario del Barrio de la Salud y del Plan de Intervención Integral del Barrio del Coll financiado por la Ley de Barrios de la Generalitat, como ejemplo de nuevas políticas de regeneración urbana para una sociedad compleja (Martí-Costa, 2009). Se implantan innovadoras acciones locales de sostenibilidad urbana en el distrito de Gràcia como son: los Puntos Limpios de Barrio para el reciclaje de los residuos de la fracción resto (el primer punto de toda la ciudad es "Tabuenca de Gràcia”), la gestión del primer huerto urbano "L'hort de l'Avi" y, finalmente, la Agenda 21 escolar en numerosas escuelas de primaria mediante la promoción de los caminos escolares, los huertos escolares o el ahorro. Pero todas estas acciones del distrito no repercuten en el barrio de Vallcarca. En el año 2002 se aprueba definitivamente la Modificación del PGM (MPGM 2002) para el sector de la avenida Vallcarca y la calle Farigola desde la plaza Lesseps hasta el puente de Vallcarca incluyendo el núcleo antiguo de Vallcarca. El planeamiento se basa sobre todo en la realización de un nuevo bulevar y viales verdes hasta el puente de Vallcarca con el consecuente derribo de prácticamente un kilómetro de edificaciones. Esto implica la concentración espacial de la máxima edificabilidad en el núcleo central del barrio de Vallcarca con nuevos edificios de promoción pública (20\% del total) para los realojados provenientes del futuro derribo del barrio frente al $80 \%$ de nueva edificación residencial de promoción privada con la consecuente futura gentrificación del barrio y la creación de nuevos viales. La Asociación de Vecinos de la Riera-Viaducto de Vallcarca ejerce como una Asociación bombillaire (se ocupa esencialmente de organizar las fiestas) y no interviene en el proceso de alegaciones al plan urbanístico por su falta de experiencia debido a la falta de memoria relacionada con las luchas vecinales. El progresivo abandono del barrio por parte de los vecinos, y por las pequeñas y medianas empresas, presionados por las inmobiliarias, genera una lenta y gradual, pero irreversible, degradación social, económica y medioambiental, atrayendo la llegada del movimiento okupa, familiarizado con este tipo de procesos urbanísticos destructivos (Stanchieri, 2011). El movimiento okupa se caracteriza por utilizar espacios abandonados para diferentes usos sin el consentimiento de la propiedad (Pruijt, 2013) y por la falta de un bien común escaso como la vivienda y los espacios de sociabilidad (Adell; Martinez, 2004).

Durante los años noventa, si se suman las casas okupadas en Gràcia y Vallcarca se pueden sumar unas cuarenta okupaciones de espacios (Blanco; Gomà, 2003). Finalmente, una parte de los vecinos con el empuje de la comunidad okupa llevan a cabo diferentes acciones reivindicativas como manifestaciones, grafitis, pancartas y crean la "Plataforma 
Salvem Vallcarca" que permite por primera vez reagrupar la diversidad y representatividad social del barrio.

\subsection{La creación de un huerto urbano como experiencia para preparar el cambio}

A partir del año 2004, la Plataforma de Vallcarca realiza un primer paso en la preparación para el cambio, sin realmente ser una acción prevista como tal, con la apropiación del espacio central del antiguo núcleo urbano ante la inexistencia histórica de espacios de encuentro para la comunidad local. Este espacio, denominado "Can Carol", tiene una idónea ubicación en el centro del barrio y una perfecta orientación sur y está compuesto por un edificio prácticamente en ruina (antigua escuela del barrio en la época republicana y adquirido por una inmobiliaria privada a finales de los noventa), un jardín romántico y una era de carácter salvaje e idílico. Esta identidad paisajística única está rodeada de árboles de gran desarrollo como el majestuoso tilo, las dos frondosas higueras, el níspero, los dos laureles y todo ello revalorizado medioambientalmente con la existencia de tres antiguos pozos de agua, como elemento característico de la antigua explotación agrícola. Se inician unos huertos urbanos (Figura 6) con dos metodologías diferentes de gestión, de un lado los huertos individuales por parte de los vecinos y, del otro, un huerto comunitario gestionado por el colectivo okupa para fomentar la cooperación con una gestión ecológica del suelo, de las plantas y del agua.

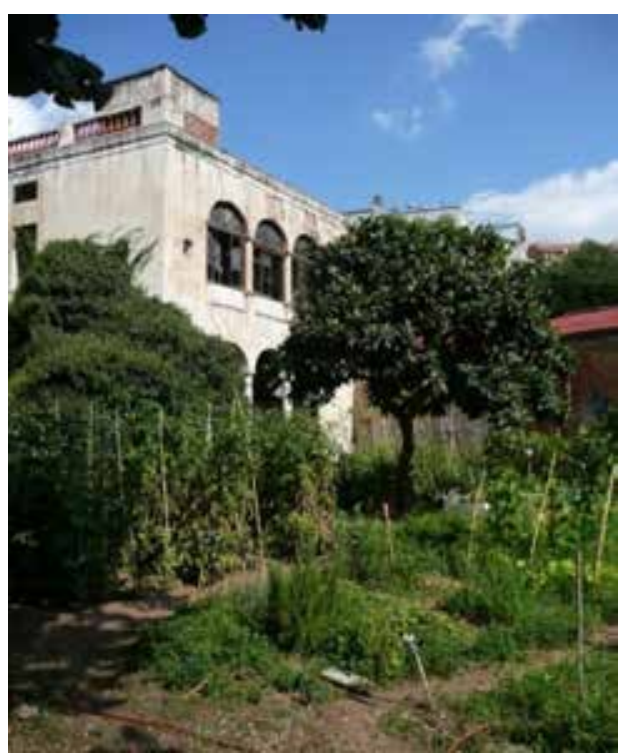
Desde 2004 hasta 2008, para-

lelamente a las actividades hortícolas, se organizan diferentes actividades abiertas a todo el barrio, como paellas populares, noches de cine con películas representativas de iniciativas colectivas como La estrategia del Caracol, y la presentación del libro El cielo está enladrillado, entre el mobbing y la violencia inmobiliaria y urbanistica ${ }^{2}$ sobre la especulación urbanística en la Barceloneta y el tema de la gentrificación. También se programan recitales poéticos amenizados por el poeta Ramón Serrano, que también estuvo involucrado en importantes reivindicaciones vecinales de su barrio natal de San Gervasio con la recuperación de los jardines de la torre Sagnier de la calle Atenas. El espacio de Can Carol, del cual un $50 \%$ corresponde a espacio público según el planeamiento, se convierte poco a poco en el nuevo espacio para la identidad social, cultural, ecológica y urbana del barrio, donde se fomentan iniciativas de sostenibilidad autoorganizadas frente a la estética de destrucción integral. Esta preparación para el cambio se construye con el liderazgo del movimiento okupa como red sombra (Olsson, 2006), genera nuevas redes de proximidad y de conexión (talleres de permacultura, documentales reivindicativos, debates urbanos, etc.) totalmente desvinculadas de las políticas oficialistas de sostenibilidad aplicadas en el distrito de

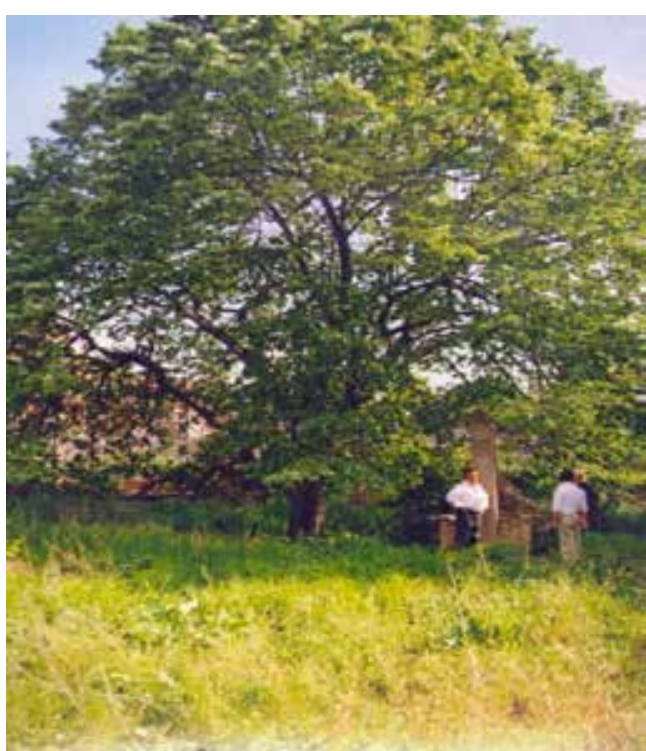

Figura 6. Huertos de "Can Carol" y los pozos de agua. Fuente: Rafael de Balanzo.

2 Ver VV.AA. (2006). El cielo está enladrillado. Entre el mobbing y la violencia inmobiliaria y urbanistica. Taller contra la Violencia Inmobiliaria y Urbanística. Barcelona: Edicions Bellaterra. Recuperado el 15 de septiembre de 2015, de: http:/ / www.edbellaterra.com/uploads/pdfs/mobbing. pdf. 
Gràcia. Una comunidad local, que se relaciona en torno a un huerto urbano, refuerza su capital social y además promueve la diversidad, la autoorganización y el aprendizaje adaptativo, todos ellos atributos necesarios de las sociedades resilientes (Tidball et al., 2007). A través de estas actividades, los usuarios crecen con múltiples competencias como gestionar un huerto, trabajar con grupos multiculturales, crear redes sociales, tener un rol activo en la seguridad del barrio hasta intervenir en los debates urbanos ante las instituciones municipales (Westphall, 2003). Según Tidball et al. (2007), la diversidad de los usuarios y de las actividades de los huertos urbanos da la oportunidad de compartir y aprender. Hoy por hoy, los huertos comunitarios son principalmente productores de convivencia, un recurso de pedagogía política y, de forma secundaria, producen verduras y hortalizas (Fernández y Moran, 2012).

\subsection{El colapso sistémico del barrio de Vallcarca}

El partido Esquerra Republicana de Catalunya, ERC, que gobierna en el distrito de Gràcia con la figura de Ricard Martínez (educador social), reconoce la mala praxis del proceso urbanístico en el último consejo de barrio de su mandato en 2007, añadido a la multitud de contenciosos jurídico-administrativos iniciados por los vecinos que llegan hasta el Tribunal Constitucional y el Parlamento Europeo. Pero a partir del 2008, coincidiendo con la llegada del nuevo regidor del distrito

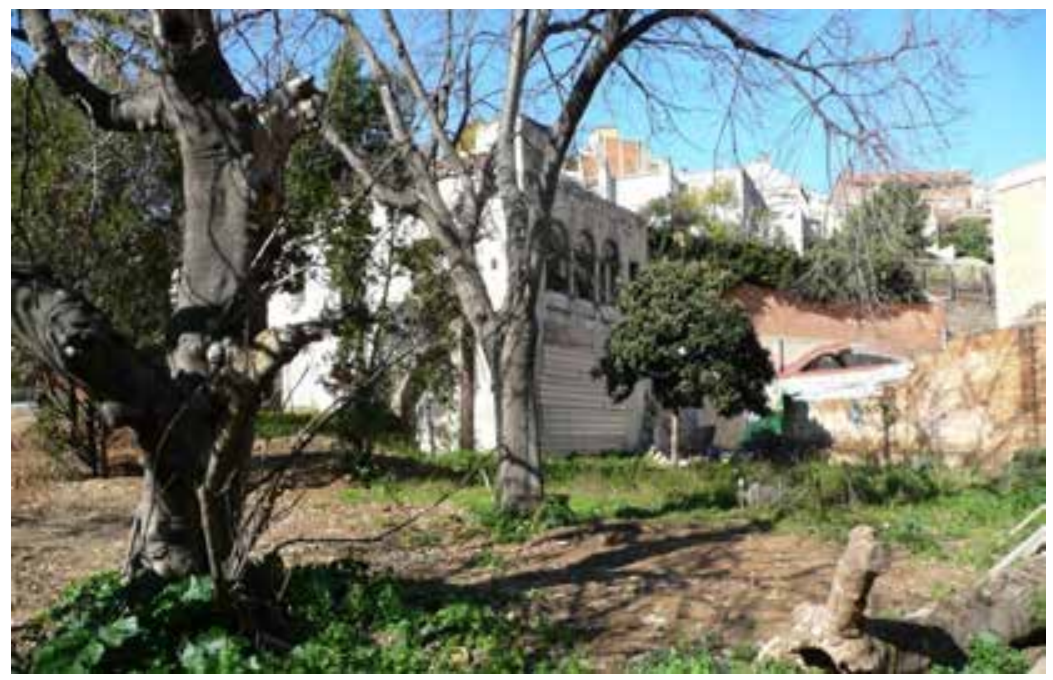
del PSC, Guillem Espriu, se aprueba el Plan de Mejora Urbana de Vallcarca y se inicia de manera contundente e imparable el derribo de la parte central del barrio. El espacio de "Can Carol" se convierte en el primer gran derribo simbólico donde se vierten decenas de toneladas de grava para cegar los pozos de agua y el terreno productivo del huerto (Figura 7). La Plataforma Salvem Vallcarca consigue salvar in extremis los árboles que son inexistentes en el diseño del futuro espacio público proyectado por la administración municipal. ${ }^{3}$ El nuevo regidor socialista de Gràcia ignora las iniciativas existentes en Can Carol y ejecuta el plan urbanístico. Los promotores inmobiliarios intensifican el proceso de derribo y destrucción integral del

Figura 7. Derribo de los huertos de Can Carol por la administración municipal. Fuente: Rafael de Balanzo.
3 Ver Resposta dels veïns $i$ de les veïnes de vallcarca al concurs del Pla buits. Recuperado el 15 de septiembre de 2015, de: http://www.rosellbernardez.com/rosell_ bernardez_P_EP.html\#farigola barrio tanto desde el punto de vista físico (con otros espacios emblemáticos como el bar del Parador con su patio de limoneros, palosantos y palmeras), como desde el social y económico (desaparecen los últimos negocios como la panadería, la tintorería, la carpintería, los centros de okupación social, etc.) y se inaugura por parte del distrito el espacio de Can Carol en mayo de 2009 como un gran espacio baldío para pasear los perros y como atajo para acceder a la boca del metro. La inexistencia de un espacio de identidad propio para el barrio y sobre todo para la Plataforma Salvem Vallcarca y el colectivo okupa, ralentiza y prácticamente anula temporalmente todas las actividades del barrio, excepto las fiestas organizadas por parte de la asociación vecinal formal. Está comprobado a partir de casos de estudios en barrios destruidos por la renovación urbana que las instituciones comunitarias y las redes sociales locales a menudo sobreviven incluso con la destrucción del pro- 
pio entorno físico (Gotham; Campanella, 2005). Por ejemplo, en un barrio de Boston derribado por las máquinas durante años se reunieron los vecinos y crearon una revista trimestral del barrio inexistente (Gotham; Campanella, 2005). La Plataforma Salvemos Vallcarca sigue la difusión mensual de artículos reivindicativos sobre el proceso urbanístico de Vallcarca (CSI Vallcarca en el diario L'Independent de Gràcia) ${ }^{4}$ y también se realiza un nuevo e importante documental cinematográfico sobre el barrio. La aparición del documental titulado Encaixonats ${ }^{5}$ (Encajonados) a principios de 2011 bajo la iniciativa de la joven e incisiva directora de cine, Marta Saleta, trata la historia del desahucio de una familia del barrio. El documental se convierte en un éxito rotundo y se multiplica su difusión por multitud de foros relacionados con los nuevos movimientos sociales urbanos de la ciudad como el Festival Internacional de Cine de la Mujer, las Asociaciones de Vecinos de la Vila de Gràcia, las entidades culturales como RAI, OACU (Observatorio de Antropología del Conflicto Urbano) de la Universidad de Barcelona, el Colegio de Arquitectos de Cataluña, ${ }^{6}$ los centros cívicos municipales y el Museo de Arte Contemporáneo de Barcelona. Esto permite la realización de apasionantes debates sobre las prácticas urbanísticas en Barcelona y sobre el barrio de Vallcarca y, por último, da paso a un proceso de empoderamiento de la comunidad local a la espera de la ventana de oportunidad para el cambio (Olsson et al., 2006).

\subsection{Vallcarca y la ventana de oportunidad}

Tras el fallido referéndum de la Diagonal y la dimisión de la regidora Itziar Gonzalez en Ciutat Vella para activar la participación ciudadana en 2010, se genera en Barcelona el evento del 15 de mayo (15-M) causado por el desapego de la sociedad a las elecciones municipales. El movimiento del 15-M se reivindica con la ocupación del espacio público para realizar sus actividades políticas y el movimiento traspasa a la escala del barrio, para reivindicar temas como la educación, la sanidad, la vivienda y el espacio público. En el caso del barrio de Vallcarca, van tomando cada vez más protagonismo las redes de proximidad y, sobre todo, las nuevas redes de conexión como la Asociación Amics del Bosc Turull, la cooperativa ecológica Valldures, Heura Negra, la Asociación Ecos, el Ateneu Popular de Vallcarca y la Candidatura de Unidad Popular (CUP), que reactivan y repiten las iniciativas anteriores con la creación intencionada de un nuevo huerto urbano en un solar contiguo a Can Carol para generar resiliencia (Figura 8). Tal como afirma Olsson (2014), cuando aparece la ventana de oportunidad, los agentes transformacionales se sitúan simultáneamente en las diferentes escalas. Al inicio del año 2012 diferentes prácticas urbanísticas coinciden simultáneamente en las tres escalas de la panarquía organizativa de la ciudad de Barcelona (ciudad, distrito y barrio), conformando relaciones de revuelta y memoria:

- En el nivel superior de la panarquía, la nueva Área de Hábitat Urbano del Ayuntamiento de Barcelona está confrontada con la unión de los técnicos de urbanismo y técnicos de la participación ciudadana (provenientes de la experiencia de la Agenda 21) en una sola área de trabajo. El nuevo Plan BUITS ${ }^{7}$ (Vacíos Urbanos con Implicación Territorial y Social) se concibe como el instrumento de cambio para el impulso de la gestión temporal de los espacios vacíos en solares públicos con la implicación ciudadana
4 Ver página web del Diari Independent de Gràcia. Recuperado el 15 de septiembre de 2015, de: http://independent.cat/ gracia/.

5 Ver el doccumental Encaixonats. Recuperado el 15 de septiembre de 2015, de: http://encaixonatsavallcarca.wordpress.com/el-documental-encaixonats/. 6 Ver La transformació de Vallcarca al cicle Ecumenòpolis. Recuperado el 15 de septiembre de 2015, de: http://www.e-coac. com/prensa/e-coac_noticies/noticies8+ . asp? Id $=942$.

7 Ver Pla BUITS. Recuperado el 15 de septiembre de 2015, de: http://www.bcn. cat/habitaturba/plabuits. 


\section{iEh, vecindario!... Ep, veïnat !.....}

\section{iUn huerto en Vallcarca! Un hort a Vallcarca!}

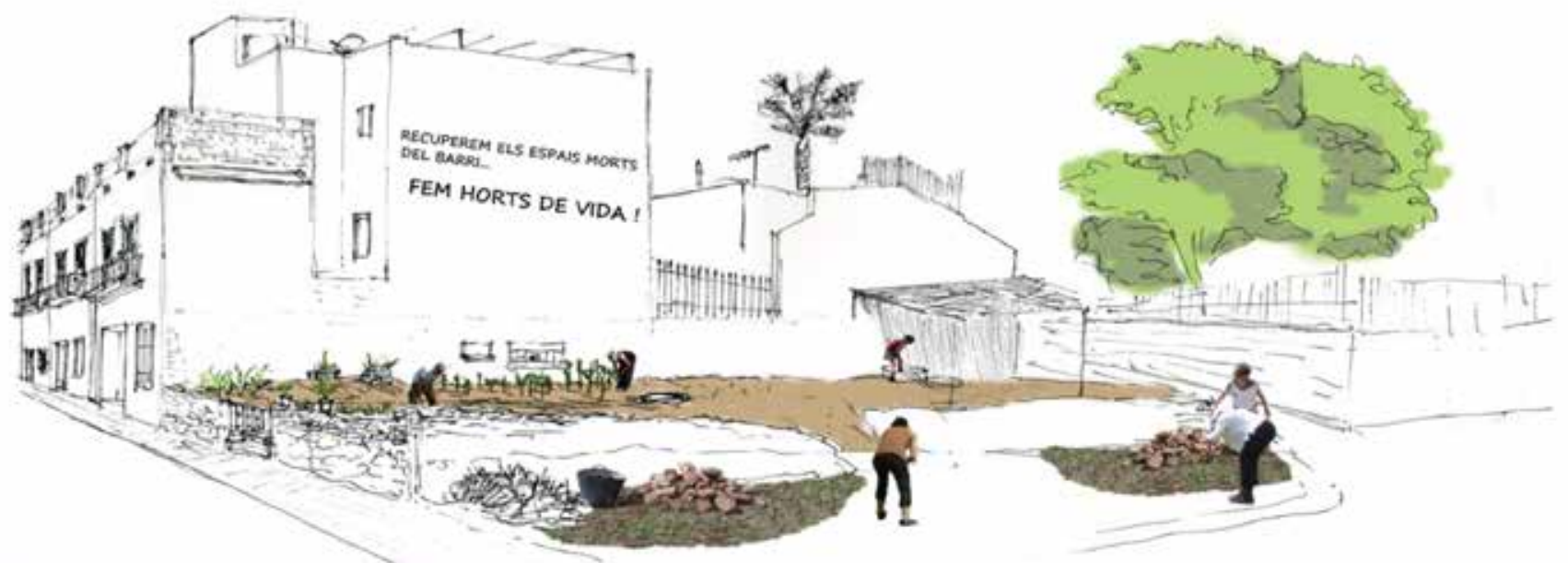

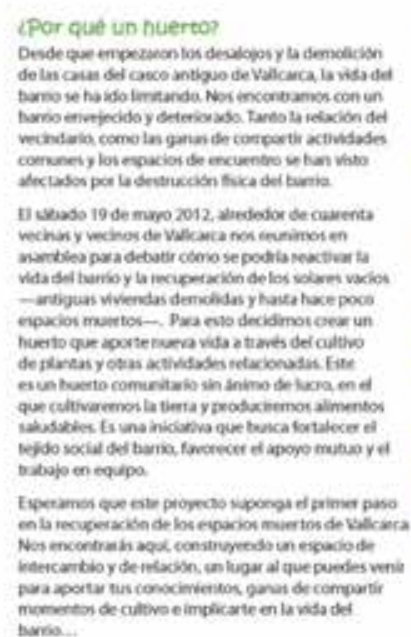

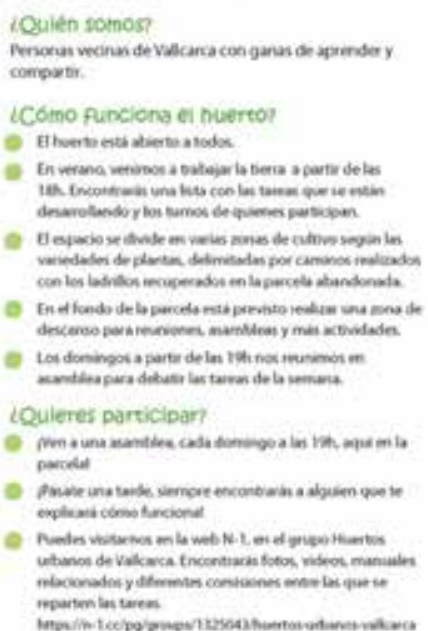

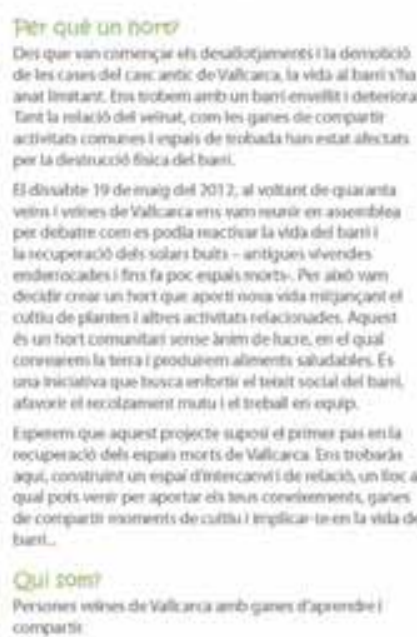

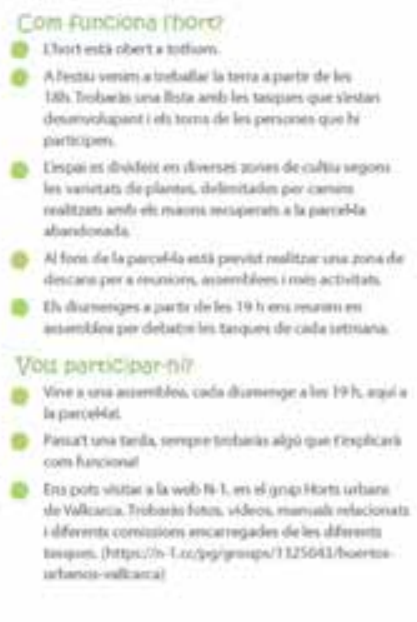

Figura 8. Cartel de los huertos de Vallcarca. Fuente: https://n-1.cc/g/huertosurbanos-vallcarca. con el lema " ¿Tienes alguna idea para dinamizar este espacio público?". El Ayuntamiento de Barcelona presenta "Can Carol" de Vallcarca como un nuevo Plan BUITS.

- En el nivel intermedio del distrito de Gràcia, bajo la iniciativa de los Servicios Sociales, se decide ceder la gestión provisional del nuevo equipamiento educativo y medioambiental del distrito situado en Vallcarca a los ciudadanos. El equipamiento, sin recursos humanos y económicos por parte de la administración, empieza a funcionar gracias a la iniciativa de la recién creada Asociación local Amics del Bosc Turull, cuya misión es fomentar iniciativas y acciones para la sostenibilidad local. La asociación se compone de un equipo mixto de vecinos, expertos académicos y técnicos sociales y de la Agenda 21 local que tienen como punto común la visión holística de los SSE y que actúan como agentes transformacionales en las diversas escalas de la administración y del barrio.

- En el nivel inferior de la panarquía, los colectivos del barrio surgidos de los nuevos movimientos sociales urbanos se unen por su lado para crear una nueva entidad llamada Asamblea Vallcarca (Figura 9) con el objetivo de construir una red de redes aún más sólida en defensa de lo común y de la identidad del ba- 
rrio, como el patrimonio cultural, medioambiental, y social. Este nueva red social compuesta de redes de proximidad y redes de conectividad presenta una contrapropuesta $^{8}$ (no normalizada según la normativa del concurso) al Plan BUITS y establece una hoja de ruta para el barrio basado en la autoorganización y la autogestión del espacio público en base a la experiencia adquirida en los últimos años de preparación al cambio. Paralelamente, la Asociación Amics del Bosc Turull conecta con los colectivos locales y la academia con el objetivo de introducir el concepto de sostenibilidad en el proceso urbanístico con la colaboración de re-

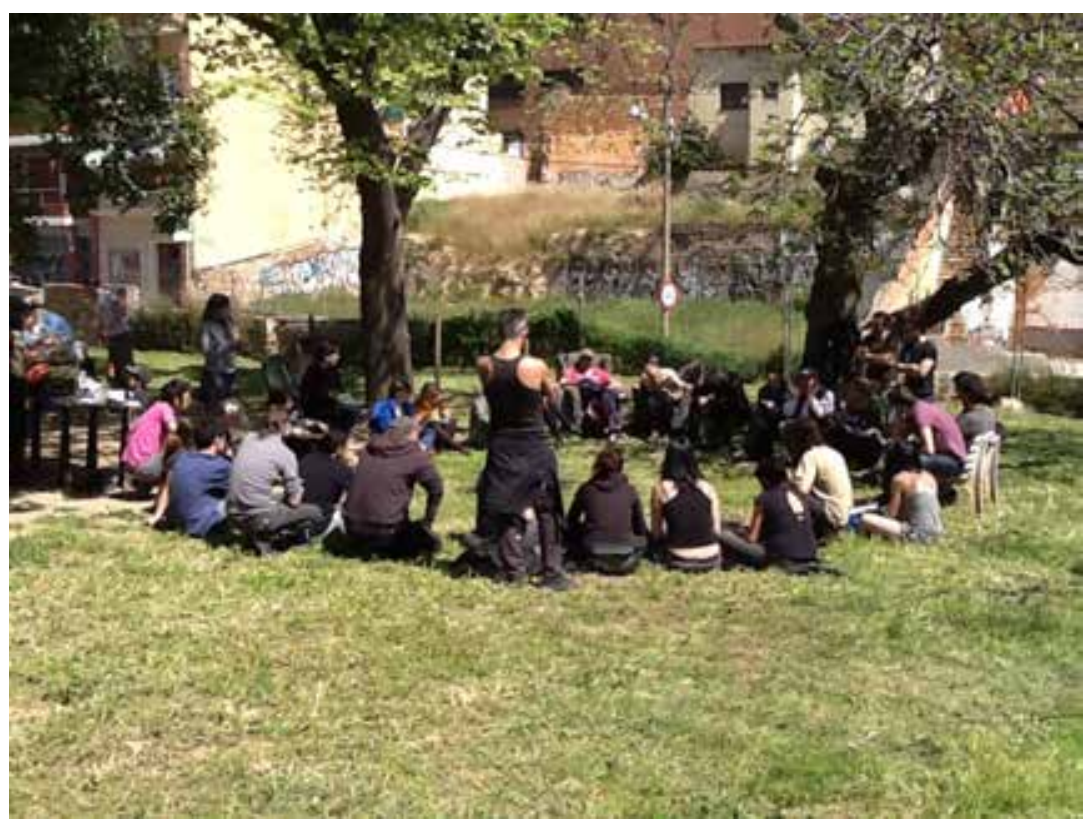
des de expertos como el Instituto de sostenibilidad de la UPC (IntraScapeLab), la Asociación de Arquitectos sin Fronteras (ASF) y el grupo de Trabajo Actions Without Borders de la Unión Internacional de Arquitectos. Pero será esencial la colaboración del movimiento de las Ciudades en Transición para crear un grupo de investigación transversal en el barrio como observatorio de la resiliencia urbana.

A pesar de la negativa burocrática del Área de Hábitat Urbano de aceptar la propuesta escrita titulada "Contrapropuesta ${ }^{9}$ del barrio de Vallcarca”, para el concurso del plan BUITS, declarando desierto el resultado para Vallcarca, el documento provoca un gran interés por parte de las nuevas redes de conexión como la Escuela de Arquitectura de Barcelona (ETSAB). ${ }^{10}$ De manera inesperada, la actividad académica de los talleres de proyectos X de la ETSAB, bajo la exigencia de los estudiantes, se traslada al propio barrio con el objetivo de ejecutar los objetivos incluidos en dicha Contrapropuesta. La participación de más de 50 alumnos, profesores y, por supuesto, de numerosos vecinos y colectivos en las sucesivas presentaciones públicas del proyecto para el espacio público de "Can Carol" permite realizar de manera autogestionada y autoconstruida el mobiliario urbano reivindicado, así como la maqueta del barrio. Estas acciones se presentan de manera espontánea en un acto multitudinario ciudadano con el apoyo del FAD (Fomento de las Artes Decorativas) (Figura 10). Dos semanas después, coincidiendo con las fiestas de Sant Joan y después de superar un sinfín de trabas administrativas por parte del distrito de Gràcia, se instala de manera definitiva el mobiliario urbano autoconstruido en "Can Carol" y se celebra nombrando el espacio de "Can Carol" como la "Plaza de la Farigola ${ }^{11}$ por parte de la comunidad local (Figura 11). Finalmente, en el equipamiento medioambiental y gestionado por la Asociación Amics del Bosc Turull, se organiza un evento para la presentación del documento de la Contrapropuesta al Plan BUITS con la maqueta del barrio como escenario y con el apoyo logístico de la red de las Ciudades en Transición como facilitador del acto en el equipamiento público de Turull. La solemnidad y el impacto mediático del acto, con la nueva maqueta del barrio como elemento simbólico, y la presencia de los agentes técnicos, políticos y ciudada-
Figura 9. Asamblea de Vallcarca en el solar de "Can Carol". Fuente: Rafael Balanzo.

8 Ver resposta dels veïns i de les veïnes de vallcarca al concurs del Pla BUITS. Recuperado el 15 de septiembre de 2015, de: https://docs.google.com/file/ d/0b5uytfraownqadjrotk2uzrznvk/edit. 9 Ver Vallcarca en moviment pels solars. Recuperado el 15 de septiembre de 2015, de: https:/ /apvallcarca.wordpress. com/2013/01/24/vallcarca-en-movimentpels-solars/.

10 Ver Arquitectes a la recerca del conflicte a la ciutat. En: http:/ /www.ara.cat/ premium/societat/Arquitectes-recercadel-conflicte-ciutat_0_941905888.html. 11 Ver Plaza de la Farigola (Vallcarca). Recuperado el 15 de septiembre de 2015 , de: http://bcncomuns.net/es/cpt/placade-la-farigola-vallcarca/. 

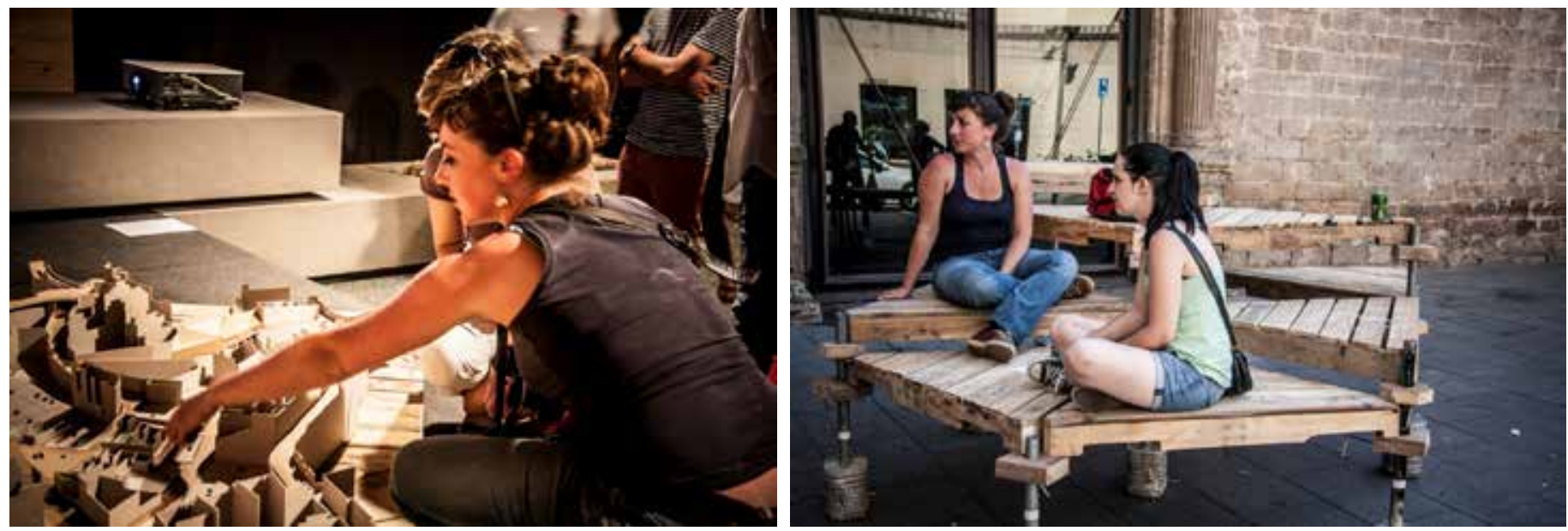

Figura 10. Arriba, exposición del mobiliario urbano y de la maqueta de Vallcarca en el FAD. Fuente: Martina Kano.

Figura 11. Inauguración de la nueva plaza Farigola en el barrio de Vallcarca. Fuente: Martina Kano.

Figura 12. Presentación de la maqueta del Barrio de Vallcarca. Fuente: Francesc Rom
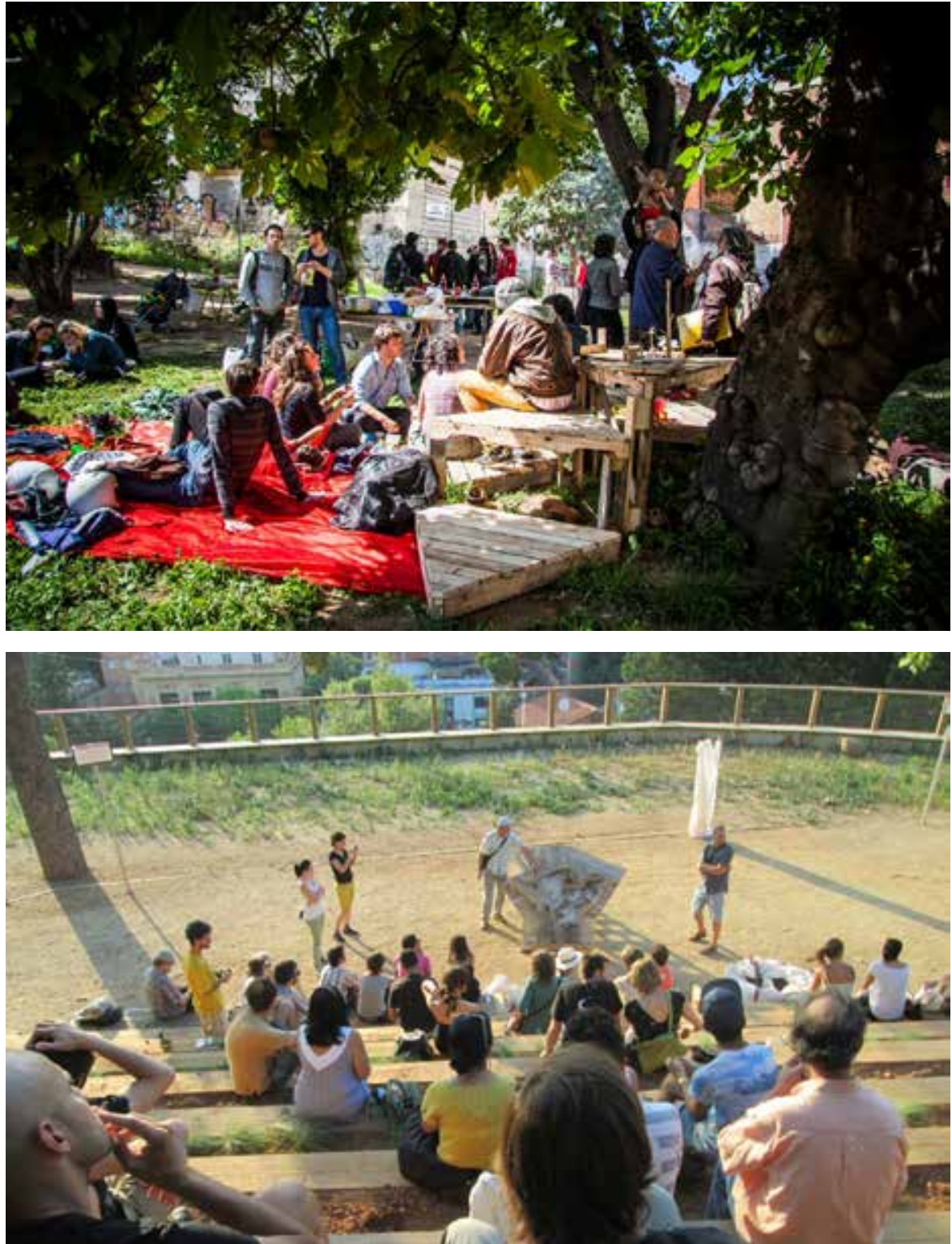

nos del Distrito de Gràcia generan y propulsan un proceso innovador de las futuras prácticas urbanísticas, así como un proceso de empoderamiento del barrio (Figura 12). Todas estas acciones emergentes iniciadas de manera multiescalar y simultáneamente en las tres escalas de la panarquía permiten la fluidez de las relaciones de Memoria y Revuelta para iniciar la fase de navegación por la transición para el cambio. Este proceso de microescala tiene algunas similitudes al cambio 
multiescalar de los años setenta pero con mayor complejidad al existir una nueva escala intermedia representada por el distrito de Gràcia, inexistente al inicio de los Ayuntamientos democráticos.

\subsection{La práctica urbanística en Vallcarca inicia una fase imprevisible Ilamada "navegando por la transición"}

Tiene lugar cuando surge la ventana de oportunidad para el cambio y comienza la segunda etapa navegando por la transición (Olsson et al., 2006). Siguiendo el símil utilizado por Olsson et al (2006), la transición de un sistema socioecológico se puede asemejar a navegar por rápidos o hacer rafting dada la incertidumbre que implica recorrer un camino por el que las reglas y los mecanismos previos ya no son válidos y es imprescindible la capacidad para improvisar y aprovechar las oportunidades que aparecen. Para iniciar el proceso de responder al cambio es esencial la mejora de la flexibilidad en la gobernanza multiescalar con la diversidad de las entidades locales y el rol clave de los agentes transformacionales en las diferentes escalas y sus interrelaciones según Ernstson et al. (2010). La ventana de oportunidad surge cuando el distrito de Gràcia decide instaurar la Mesa de las Entidades Locales (propulsada por la continua e insistente acción del técnico de barrio durante meses) formada por la máxima representatividad y diversidad social del barrio de Vallcarca. Esta mesa tiene por objetivo dibujar un nuevo escenario de convivencia para el barrio, con la participación de todas las redes sombra y de las entidades informales. Según Westley et al. (2013), una vez se ha instaurado una plataforma que focaliza las acciones colectivas y los recursos, las instituciones emprendedoras pueden empezar a construir una red de creatividad de ideas y acciones. Olsson et al. (2006) afirman que los factores claves para la emergencia del liderazgo de los actores claves y de las redes sombra para construir la resiliencia son:

- Reconceptualizar conceptos.

- Generar e integrar puntos de vista, ideas y soluciones.

- Comunicar e implicar personas claves de diferentes sectores.

- Moverse a diferentes escalas de la gobernanza.

- Promover y apadrinar experimentos a pequeña escala.

- Reconocer y aprovechar las ventanas de oportunidad.

- Promover novedad combinando diferentes experiencias, redes, memorias colectivas.

Para iniciar el camino para la construcción de la resiliencia, la Mesa de las Entidades del barrio de Vallcarca apuesta (incluye la administración municipal del distrito y de Hábitat Urbano) por un innovador proceso de prácticas urbanísticas (Figura 13) que no será liderado por la administración, sino por un grupo de consultores externos integrado por el grupo de trabajo de Action Without Borders de la Unión Internacional de Arquitectos ${ }^{12}$ y de la Asociación Arquitectos sin Fronteras con gran experiencia en fomentar la resiliencia urbana en contextos urbanos degradados como son los barrios de la llamada ciudad informal, exis-
Figura 13. Póster del Taller de Participación Ciudadana. Fuente: Assemblea de Vallcarca.

12 Ver AWB-UIA Work Programme. Recuperado el 15 de septiembre de 2015, de: https://awbuia.wordpress.com/. 
tentes en ciudades de Latinoamérica y África. Estas iniciativas provocan turbulencias y revuelta (Westley et al., 2013) en las institucionalizadas políticas urbanas del distrito de Gràcia y de la ciudad.

\section{Conclusión}

\subsection{Los fenómenos de revuelta y memoria y el rol de los agentes transformacionales en el barrio de Vallcarca}

La lectura de los sistemas urbanos como SSE pone en evidencia, por un lado, el rol clave de los pequeños colectivos en la evolución de las prácticas urbanísticas gracias a su flexibilidad y su autoorganización en el aumento de la resiliencia urbana local. Y por otro lado, surge la labor de la gestión social y del hábitat por parte de los agentes claves y transformacionales como facilitadores en las relaciones interescalares y extraescalares de revuelta y memoria de la panarquía. En el barrio de Vallcarca se inicia a finales de los años noventa una fase opaca del ciclo adaptativo con la implementación de un planeamiento urbanístico con el objetivo de construir un nuevo barrio residencial de medio y alto standing. Esta etapa de empobrecimiento muy institucionalizada y rígida con recursos económicos y con la memoria de la época autoritaria municipal del Porciolismo se centra en el derribo integral del SSE existente de Vallcarca. A partir del año 2004 las acciones autoorganizadas de aprendizaje facilitado por las nuevas entidades transformacionales como la Plataforma Salvem Vallcarca y posteriormente la Asamblea Vallcarca y la Asociación de Amics del Bosc Turull provocan procesos de memoria y revuelta que introducen innovación y creatividad hacia las escalas superiores de la Administración. Las acciones emergentes del barrio de Vallcarca adquieren ventaja en el momento que se presenta la oportunidad para el cambio (Westley et al., 2013), se anticipan al colapso de la Administración Municipal ante la crisis actual y se convierten en los futuros cimientos del refuerzo de la resiliencia local (Olsson et al., 2006).

\subsection{Los atributos de la resiliencia urbana}

En el barrio de Vallcarca, como en la evolución de los SSE, son sobre todo cuatro los factores que hacen que la comunidad local sea más resiliente y facilite su supervivencia en el tiempo. Según Berkes et al. (2003) son los siguientes atributos que de forma simultánea se manifiestan para garantizar dicha sostenibilidad:

- Los cambios, las presiones y la devastación deben ser considerados como una fuente de aprendizaje para la transformación del SSE.

- La diversidad sistémica provee las bases para las futuras respuestas adaptativas.

- El conocimiento y la habilidad del SSE desarrolla e incrementa la capacidad de aprender, innovar y adaptarse.

- El nivel de autoorganización y autosuficiencia es básico para el proceso.

Los pequeños colectivos del barrio de Vallcarca, más flexibles y con un alto conocimiento de los retos locales, gracias al aprendizaje para 
adaptarse a los diferentes colapsos y crisis sobrevenidas durante estos últimos años en el contexto local, consiguen reorganizarse y emerger en una nueva red de iniciativas culturales, medioambientales y económicas autoorganizadas, diversas, autosuficientes, innovadoras y creativas y reforzar la nueva identidad cultural del barrio. Según afirman Gotham y Campanella (2010), las comunidades resilientes no retornan a un estado de precrisis, sino que tienen la capacidad de reinventarse ellas mismas con nuevas relaciones, modos de organización y creación de redes. La capacidad de una ciudad para adaptarse descansa en gran parte en sus ciudadanos, y si los ciudadanos llegan a faltar, la ciudad perdería su oportunidad (Campanella, 2005).

Para este artículo quiero agradecer la colaboración de todos los vecinos de Vallcarca y colectivos que han compartido este proceso durante todos estos años, y en particular a Jordi Balari y Pedro Lorenzo, miembros del grupo AWB de la Unión Internacional de Arquitectos y a Francesc Magrinya del Instituto de Sostenibilidad de la UPC por la ayuda aportada. 


\section{Referencias}

Adger, W. (2000). Social and ecological resilience: are they related? Progress in human geography, 24 (3).

Aricó, G.; Stanchieri, M. L. (2013). La trampa urbanística de los 'vacíos urbanos': casos etnográficos en Barcelona. En DAROQUI A.(Comp.) X Jornadas de Sociología de la UBA, 2013.

Berkes, F. et al. (2003). Navigating social-ecological systems: building resilience for complexity. Cambridge University Press.

Blanco, I.; Gomà, R. (2003). Gobiernos locales y redes participativas: retos e innovaciones. Reforma y Democracia, 26, p. 75-100.

Borja, J. (2009). Luces y sombras del urbanismo de Barcelona. Editorial UOC.

Busquets, J. (2005). The urban evolution of a compact city. Rovereto: Nicolodi.

Campanella, T.J. (2006). Urban resilience and the recovery of New Orleans. Journal of the American Planning Association, 72 (2), p. 141-146.

Capel, H. (2005). El modelo Barcelona: un examen crítico. Ed. del Serbal.

. (2010). Epílogo: ¿En qué ha fallado Barcelona? Finisterra-Revista Portuguesa de Geografia, 90, p. 173-204.

Dalmau, M. (2010). La Colònia Castells: un barri al corredor de la mort. Quaderns-e de l'Institut Català d'Antropologia, 15, p. 141-160.

Davoudi, S.; Brooks, E.; Mehmood, A. (2013). Evolutionary resilience and strategies for climate adaptation. Planning Practice E Research, 28 (3), p. 307322.

Delgado, M. (2007). La ciudad mentirosa: fraude y miseria del "modelo Barcelona". Los libros de la Catarata.

De Manuel, E. (2010). Construyendo triángulos para la gestión social del hábitat. Habitat y sociedad, 1, p. 13-37.

Ernstson, H., et al. (2010). Scale-crossing brokers and network governance of urban ecosystem services: The case of Stockholm. Ecology and Society, 15 (4), p. 28.

FAVB. (2009). 40 anys d'acció veïnal, El carrer, 114.

Fernández De Casadevante, J. L.; Moran Alonso, N. (2012). Cultivar la resiliencia. Los aportes de la agricultura urbana a las ciudades en transición. $P a$ peles de relaciones ecosociales y cambio global.

Fernández Durán, R. (2006). El tsunami urbanizador español y mundial: sobre sus causas y repercusiones devastadoras, y la necesidad de prepararse para el previsible estallido de la burbuja inmobiliaria. Barcelona: Virus Editorial.

Folke, C. (2006). Resilience: The emergence of a perspective for social-ecological systems analyses. Global environmental change, 16 (3), p. 253-267.

Folke, C; Colding, J; Berkes, F. (2003). Synthesis: buil- ding resilience and adaptive capacity in social-ecological systems. En: Navigating social-ecological systems: Building resilience for complexity and change, $\mathrm{p}$. 352-387.

Gotham, K. E.; Campanella, R. (2011). Coupled vulnerability and resilience: The dynamics of cross-scale interactions in post-Katrina New Orleans. Ecology and Society, 16 (3), p. 12.

Gunderson, L. (2010). Ecological and human community resilience in response to natural disasters. Ecology and Society, 15 (2), 18.

Holling, C. S. (1973). Resilience and stability of ecological systems. Annual review of ecology and systematics, p. 1-23.

_. (1996). Engineering resilience versus ecological resilience. Engineering within ecological constraints, p. 31-44.

- (2001). Understanding the complexity of economic, ecological, and social systems. Ecosystems, 4 (5), p. 390-405.

Holling, C. S.; Gunderson, L. H.; Peterson, G. D. (2002). Sustainability and panarchies. En: Panarchy: Understanding transformations in human and natural systems, p. 63-102. Island Press.

Hopkins, R. (2008). The transition handbook: from oil dependency to local resilience. Totnes: Green books.

_. (2011). The transition companion: Making your community more resilient in uncertain times. Chelsea Green Publishing.

Lees, L.; Slater, T.; Wyly, E. (2013). Gentrification. Routledge.

Martí-Costa, M. (2009). La participació Ciutadana en Urbanisme: els Plans d'Ordenació Urbanística Municipal (Informe de investigación). Barcelona: Institut de Govern i Polítiques Públiques.

Martínez, M. (2004). Del urbanismo a la autogestión: una historia posible del movimiento de okupación en España. En Adell, R. y Martínez, M. En: ¿̨Dónde están las llaves? El movimiento okupa: prácticas y contextos sociales. Los Libros de la Catarata, p. 61-88.

Montaner, J. M. (2004). La evolución del modelo Barcelona (1979-2002). Urbanismo en el siglo XXI: una visión crítica. Bilbao, Madrid, Valencia, Barcelona. Barcelona: Edicions UPC, ETSAB (Arquitext), p. 203219.

Olsson, P. et al. (2006). Shooting the rapids: navigating transitions to adaptive governance of social-ecological systems. Ecology and society, 11 (1), p. 18.

Pickett, S. T.A.; Cadenasso, M. L.; Grove, J. M. (2004). Resilient cities: meaning, models, and metaphor for integrating the Ecological, socio-economic, and planning realms. Landscape and urban planning, 69 (4), p. 369-384.

Portugali, J. et al. (2012). Complexity theories of cities have 
come of age: an overview with implications to urban planning and design. Springer.

Pruijt, H. (2013). The logic of urban squatting. International Journal of Urban and Regional Research, 37 (1), p. 19-45.

Stanchieri, M. (2011). Retóricas y prácticas de una transformación urbanística: El caso de Vallcarca en Barcelona, Revista Diagonal, 32. Recuperado el 15 de septiembre de 2015, de: http:/ / www.revistadiagonal.com/author/stanchieri/

Tatjer, M. Killinger, C. L.(2010). Barraques: la Barcelona informal del segle XX. MUHBA, Museu d'Història de Barcelona.

Tidball, K. G.; Krasny, M. (2007). E. From risk to resilience: What role for community greening and civic ecology in cities. Social learning towards a more sustainable world, p. 149-164.

Walker, B., et al. (2004). Resilience, adaptability and transformability in social-ecological systems. Ecology and society, 9 (2), p. 5.

Walker, B.; Salt, D. (2012). Resilience practice: building capacity to absorb disturbance and maintain function. Island Press.

Westphal, L.(2003). M. Urban greening and social benefits: a study of empowerment outcomes. Journal of Arboriculture, 29 (3), p. 137-147.

Westley, F. R., et al. (2013). A theory of transformative agency in linked social-ecological systems. Ecology and Society, 18 (3), p. 27.

BALANZO JOUE, Rafael de. Barcelona, caminando hacia la resiliencia urbana en el barrio de Vallcarca. Hábitat y Sociedad, 2015, n. ${ }^{\circ} 8$, pp. $75-92$.

$<$ www.habitatysociedad.us.es $>$

http://dx.doi.org/10.12795/HabitatySociedad.2015.i8.04

$\bigcirc$ 